\title{
TAUTOLOGICAL ALGEBRA OF THE MODULI SPACE OF SEMISTABLE BUNDLES ON AN ELLIPTIC CURVE
}

\author{
ARIJIT MUKHERJEE
}

\begin{abstract}
In this paper, our aim is to find the relations amongst the cohomology classes of Brill-Noether subvarieties of the moduli space of semistable bundles over an elliptic curve. We obtain results similar to the Poincaré relations on a Jacobian variety.
\end{abstract}

\section{Contents}

1. Introduction

2. Notations

3. Brill-Noether loci of semistable bundles over an elliptic curve

4. Tautological algebra of semistable bundles of degree zero over an elliptic curve

5. Main theorems

Acknowledgement

References

\section{Introduction}

Suppose $C$ is a smooth projective variety of genus $g$, defined over the complex numbers. The Picard variety $\operatorname{Pic}^{d}(C)$ is the moduli space of isomorphism classes of line bundles of degree $d$ on $C$. For $1 \leq i \leq g-1$, there are naturally defined subvarieties, namely the Brill-Noether subvarieties $W_{i}$ of $\operatorname{Pic}^{i}(C)$, which parametrize line bundles $L$ with $h^{0}(L) \geq 1$. Let $\Theta$ denote the theta divisor $W_{g-1} \subset \operatorname{Pic}^{g-1}(C)$. Choosing isomorphisms $\operatorname{Pic}^{i}(C) \simeq J(C)$, the classical Poincaré relations determine the relations between the cohomological classes of $W_{i}$, in $J(C)$ :

$$
\left[W_{i}\right]=\frac{1}{(g-i) !}[\Theta]^{g-i} \in H^{*}(J(C), \mathbb{Q}) .
$$

See [1, Ch 1, §5, p. 25].

Our aim in this paper is to investigate relations between the cohomology classes of the Brill-Noether subvarieties in the moduli space of higher rank vector bundles on a curve. Brill-Noether subvarieties are more subtle in this situation and not much seems to be known in this direction. We consider genus one case in this paper.

Let $E$ denote a complex elliptic curve. The moduli space of semistable bundles over $E$ has been computed by M. F. Atiyah [2]. L. Tu [6] explicitly described the Brill-Noether

\footnotetext{
${ }^{0}$ Mathematics Classification Number: 14D20, 14H40, 14H52, 14F25.

${ }^{0}$ Keywords: Jacobian varieties, Elliptic curves, vector bundles, moduli spaces.
} 
subvarieties in this moduli space. In this paper, a tautological class is the cohomology class of Brill-Noether subvariety in the cohomology ring and the subalgebra of the cohomology ring generated by those tautological classes is called tautological algebra. We describe the relations amongst the tautological classes in the cohomology ring of the moduli space in this paper.

We denote by $f$ the strong equivalence class of $F$, a vector bundle over $E$, in the sense of [5, $\S 8$, p. 333]. Let $\mathcal{M}_{E}(r, d)$ be the moduli space of strong equivalence classes of semistable bundles of rank $r$ and degree $d$ over $E$. Let $X$ be a subvariety of $\mathcal{M}_{E}(r, d)$ (or of $\mathcal{S U}_{E}(r, L)$ ), then by $[X]$ we denote the cohomology class of $X$ in $H^{*}\left(\mathcal{M}_{E}(r, d)\right)$ (respectively in $\left.H^{*}\left(\mathcal{S U}_{E}(r, L)\right)\right)$.

By [6], we note that the Brill-Noether loci are trivial for positive degree vector bundles (either empty or the whole moduli space), and for line bundles of degree 0 (either empty or singleton). Therefore, we consider the Brill-Noether loci when degree of a vector bundle is 0 and the rank is more than 1 . Let $L$ be a line bundle of degree 0 and let $i$ be any non-negative integer. The Brill-Noether loci inside $\mathcal{S U}_{E}(r, L)$ are defined as follows:

$$
W_{r, L}^{i}(\exists):=\left\{f \in \mathcal{S U}_{E}(r, L) \mid h^{0}(F) \geq i+1 \text { for some } F \in f\right\} .
$$

We denote by $\left[W_{r, L}^{i}(\exists)\right]$, a tautological class in $H^{*}\left(\mathcal{S U}_{E}(r, L), \mathbb{Z}\right)$. We also consider the Brill-Noether loci, denoted by $W_{r, 0}^{i}(\exists)$, inside $\mathcal{M}_{E}(r, 0)$, defined as follows:

$$
W_{r, 0}^{i}(\exists):=\left\{f \in \mathcal{M}_{E}(r, 0) \mid h^{0}(F) \geq i+1 \text { for some } F \in f\right\} .
$$

(See [6]).

In \$5, we prove the main theorems on the relations amongst the tautological classes in $H^{*}\left(\mathcal{S U}_{E}(r, L), \mathbb{Z}\right)$ and in $H^{*}\left(\mathcal{M}_{E}(r, 0), \mathbb{Z}\right)$. We show:

Theorem 1.1. Let $r$ be any positive integer and let $L$ be a degree 0 line bundle over $E$. Then $W_{r, L}^{0}(\exists)$ is a divisor inside $\mathcal{S U}_{E}(r, L)$. Moreover, in $H^{*}\left(\mathcal{S U}_{E}(r, L), \mathbb{Z}\right)$ we have,

$$
\left[W_{r, L}^{i}(\exists)\right]=\left[W_{r, L}^{0}(\exists)\right]^{i+1}
$$

for all $0 \leq i \leq r-2$ and the tautological algebra of $\mathcal{S U}_{E}(r, L)$ is $\mathbb{Z}[\zeta] /\left(\zeta^{r}\right)$, where $\zeta$ is the cohomology class of $W_{r, L}^{0}(\exists)$ in $H^{*}\left(\mathcal{S U}_{E}(r, L), \mathbb{Z}\right)$.

Furthermore, we show that the relations amongst $\left[W_{r, 0}^{i}(\exists)\right]$ 's in $H^{*}\left(\mathcal{M}_{E}(r, 0), \mathbb{Z}\right)$, is similar to (11). In particular, we show:

Theorem 1.2. The tautological algebra of $\mathcal{M}_{E}(r, 0)$ is

$$
H^{*}(E) \otimes \mathbb{Z}(\xi) /\left(\xi^{r}\right)
$$

Here $\xi$ is the cohomology class of the divisor $W_{r, 0}^{0}(\exists)$ on $\mathcal{M}_{E}(r, 0)$ in $H^{*}\left(\mathcal{M}_{E}(r, 0), \mathbb{Z}\right)$.

It is interesting to investigate if similar relations hold in higher genus case.

\section{Notations}

All the varieties are defined over the complex numbers.

1) $\mathcal{S U}_{E}(r, L)$ denotes the moduli space of strong equivalence classes of semistable bundles of rank $r$ and fixed determinant $L$ of degree $d$ over $E$. 
2) $S^{n} E$ is the $n$th symmetric product of the curve $E$.

3) $J(E)$ is the Jacobian variety of isomorphism classes of line bundles of degree 0 over $E$.

4) $J_{d}(E)$ is the isomorphism classes of line bundles of degree $d$ over $E$.

$5) \operatorname{det} F$ is the determinant bundle of a semistable vector bundle $F$ over $E$.

6) $\mathcal{O}(D)$ denotes the line bundle corresponding to a divisor $D$ on $E$.

7) $\mathcal{O}_{E}$ is the trivial line bundle over $E$.

8) $h^{i}(E, F)$ (or $\left.h^{i}(F)\right)$ denotes the dimension of $H^{i}(E, F)$ for a semistable bundle $F$ over E.

9) By $H^{*}(X)$ we mean $H^{*}(X, \mathbb{Z})$, the cohomology ring of a complex variety $X$ with integral coefficients.

\section{BRILl-Noether lOCI OF SEMistable BUNDLES OVER AN ELLIPTIC CURVE}

3.1. Bundles with positive degree. In this section we recall a few results from [6] which will be relevant for the next section. The following lemma is proved in [6]. For the sake of completeness we include the lemma with proof here.

Lemma 3.1. [6, Lemma 17, p. 13] Any semistable bundle $F$ of positive degree over $E$ is non-special, that is, $h^{1}(F)=0$.

Proof. Let $F$ be a semistable vector bundle of degree $d>0$ and $K_{E}$ be the canonical line bundle over $E$. Then $K_{E} \cong \mathcal{O}_{E}$ as $E$ is an elliptic curve. By Serre duality we have

$$
h^{1}(F)=h^{0}\left(K_{E} \otimes F^{*}\right)=h^{0}\left(F^{*}\right)
$$

As $F^{*}$ is also a semistable bundle and of negative degree, $h^{0}\left(F^{*}\right)=0$. Therefore, $h^{1}(F)=0$. Moreover by Riemann-Roch theorem, $h^{0}(F)=d$.

Remark 3.2. A consequence of Lemma 3.1 is that the map

$$
\begin{aligned}
h^{0}: \mathcal{M}_{E}(r, d) & \longrightarrow \mathbb{Z}_{+} \cup\{0\} \\
f & \mapsto h^{0}(F)
\end{aligned}
$$

is well defined for $d>0$, and is the constant function $d$.

Definition 3.3. Let $d>0$ and $i \geq 0$ be any two integer. The Brill-Noether loci are defined by

$$
W_{r, d}^{i}:=\left\{f \in \mathcal{M}_{E}(r, d) \mid h^{0}(F) \geq i+1\right\} .
$$

This definition is well defined by Remark 3.2 .

The following lemma is a direct consequence of Lemma 3.1, See [6, p. 13].

Lemma 3.4. Let $d>0$. Then

$$
W_{r, d}^{i} \cong \begin{cases}\emptyset & \text { if } 1 \leq d \leq i \\ \mathcal{M}_{E}(r, d) & \text { if } d \geq i+1\end{cases}
$$

Therefore Brill-Noether loci inside $\mathcal{M}_{E}(r, d)$ are not of much interest when $d>0$. Moreover for degree 0 line bundles over $E$ we have the following result. 
Lemma 3.5. The Brill-Noether loci for $d=0, r=1$ are

$$
W_{1,0}^{i} \cong \begin{cases}\emptyset & \text { if } 1 \leq i \\ \left\{\mathcal{O}_{E}\right\} & \text { if } i=0\end{cases}
$$

Proof. See [6, p. 13]. As $h^{0}(L)=0$ or 1 for a line bundle $L$ of degree zero over $E$ and moreover $h^{0}(L)=1$ if and only if $L \cong \mathcal{O}_{E}$.

3.2. Degree zero bundles. When $d=0, h^{0}: \mathcal{M}_{E}(r, 0) \longrightarrow \mathbb{Z}_{+} \cup\{0\}$ is not well defined. For example, let $F_{2}$ be the Atiyah's indecomposable bundle of rank 2 and $I_{2}$ be the trivial bundle of rank 2. Then $F_{2} \cong I_{2}$, but $h^{0}\left(F_{2}\right)=1 \neq 2=h^{0}\left(I_{2}\right)$, [2, proof of Theorem 5, p. 432].

In this case, Brill-Noether loci inside $\mathcal{S U}_{E}(r, L)$ and $\mathcal{M}_{E}(r, 0)$ are defined, see [ㅁ, p. 5]. Here $L$ is a line bundle on $E$ of degree 0 .

\section{Definition 3.6.}

$$
\begin{aligned}
& W_{r, L}^{i}(\exists):=\left\{f \in \mathcal{S U}_{E}(r, L) \mid h^{0}(F) \geq i+1 \text { for some } F \in f\right\} . \\
& W_{r, 0}^{i}(\exists):=\left\{f \in \mathcal{M}_{E}(r, 0) \mid h^{0}(F) \geq i+1 \text { for some } F \in f\right\}
\end{aligned}
$$

We have the equality:

$$
W_{r, L}^{i}(\exists)=W_{r, 0}^{i}(\exists) \cap \mathcal{S U}_{E}(r, L) .
$$

We now have the following isomorphism of moduli spaces.

Proposition 3.7. Let $r>1$ be a positive integer and $L$ a degree 0 line bundle over $E$. Then we have the isomorphisms:

$$
\begin{aligned}
\mathcal{M}_{E}(r, 0) & \cong S^{r} E \\
\mathcal{S} \mathcal{U}_{E}(r, L) & \cong \mathbb{P}^{r-1} \\
J(E) & \cong E \\
W_{r, 0}^{i}(\exists) & \cong S^{r-i-1} E \\
W_{r, L}^{i}(\exists) & \cong \mathbb{P}^{r-i-2} .
\end{aligned}
$$

Proof. See [6, Theorem 2, 3, 4 and 5; Corollary 15; p. 4-5, 10].

\section{Tautological algebra of Semistable Bundles of Degree Zero over an ELLIPTIC CURVE}

Consider the map:

$$
\begin{aligned}
\pi: J(E) \times \mathcal{S U}_{E}(r, L) & \rightarrow \mathcal{M}_{E}(r, d) \\
(l, F) & \mapsto l \otimes F .
\end{aligned}
$$

See [3, p. 338] and [4, p. 348].

This map as in (2) suggests that the cohomology subalgebra generated by $\left[W_{r, 0}^{i}(\exists)\right]$ 's is the same as that generated by $\left[W_{r, L}^{i}(\exists)\right]$ 's with coefficients lying in $H^{*}(J(E))$. We make this precise in the next section. 
Let det $: \mathcal{M}_{E}(r, 0) \rightarrow J(E)$ be the determinant map which sends $F$ to $\operatorname{det} F$ and $\alpha: S^{r} E \rightarrow$ $J_{r}(E)$ be the Abel-Jacobi map defined as $x_{1}+x_{2}+\cdots+x_{r} \mapsto \mathcal{O}\left(x_{1}+x_{2}+\cdots+x_{r}\right)$. Then we have the following commutative diagram ([6, p. 12]), which says that the determinant map and the Abel-Jacobi map can be identified.

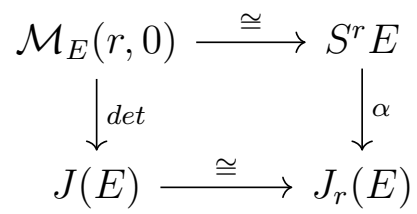

Remark 4.1. By Abel's theorem ([1, p. 18]), fiber of the map $\alpha$ over any line bundle $L \in J_{r}(E)$ is the complete linear system $|D|$ of a divisor $D$ on $E$ with $\mathcal{O}(D)=L$. Now if $r>0$, then by Serre duality $h^{1}(E, \mathcal{O}(D))=0$ and by Riemann-Roch theorem $h^{0}(E, \mathcal{O}(D))=r$. Therefore each fiber of the map $\alpha: S^{r} E \rightarrow J_{r}(E)$ is isomorphic to $\mathbb{P}^{r-1}$ if $r>0$. This is also another reason to work in $\mathcal{M}_{E}(r, 0)$ with $r>0$.

Let det $: W_{r, 0}^{i}(\exists) \rightarrow J(E)$ be the restriction of the determinant map det $: \mathcal{M}_{E}(r, 0) \rightarrow$ $J(E)$. Then we have the following commutative diagram ([, p. 16]) similar to (3) $)$.

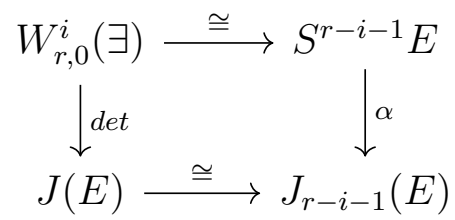

Here we assume $0 \leq i \leq r-2$. So by Remark 4.1, the fiber of $\alpha$ is isomorphic to $\mathbb{P}^{r-i-2}$.

\section{MAIN THEOREMS}

In this section we define the tautological subalgebra of $H^{*}\left(\mathcal{M}_{E}(r, 0), \mathbb{Z}\right)$ and $H^{*}\left(\mathcal{S U}_{E}(r, L), \mathbb{Z}\right)$ and prove our main theorems.

Definition 5.1. The cohomology classes $\left[W_{r, 0}^{i}(\exists)\right] \in H^{*}\left(\mathcal{M}_{E}(r, 0), \mathbb{Z}\right)$ are called the tautological classes. The subalgebra of $H^{*}\left(\mathcal{M}_{E}(r, 0), \mathbb{Z}\right)$ generated by these tautological classes is called the tautological subalgebra of $H^{*}\left(\mathcal{M}_{E}(r, 0), \mathbb{Z}\right)$.

Definition 5.2. Let $L$ be a degree zero line bundle over $E$. Then the cohomology classes $\left[W_{r, L}^{i}(\exists)\right] \in H^{*}\left(\mathcal{S U}_{E}(r, L), \mathbb{Z}\right)$ are called the tautological classes. The subalgebra of $H^{*}\left(\mathcal{S U}_{E}(r, L), \mathbb{Z}\right)$ generated by these tautological classes is called the tautological subalgebra of $H^{*}\left(\mathcal{S U}_{E}(r, L), \mathbb{Z}\right)$.

Following theorem shows that the tautological class $\zeta:=\left[W_{r, L}^{0}(\exists)\right]$ is the generator of the tautological subalgebra of $H^{*}\left(\mathcal{S U}_{E}(r, L), \mathbb{Z}\right)$.

Theorem 5.3. Let $r$ be any positive integer and let $L$ be a degree 0 line bundle over $E$. Then $W_{r, L}^{0}(\exists)$ is a divisor inside $\mathcal{S U}_{E}(r, L)$. Moreover, in $H^{*}\left(\mathcal{S U}_{E}(r, L), \mathbb{Z}\right)$ we have,

$$
\left[W_{r, L}^{i}(\exists)\right]=\left[W_{r, L}^{0}(\exists)\right]^{i+1}
$$


for all $0 \leq i \leq r-2$ and the tautological algebra of $\mathcal{S U}_{E}(r, L)$ is $\mathbb{Z}[\zeta] /\left(\zeta^{r}\right)$, where $\zeta$ is the cohomology class of $W_{r, L}^{0}(\exists)$ in $H^{*}\left(\mathcal{S U}_{E}(r, L), \mathbb{Z}\right)$.

Proof. We have the following stratification inside $\mathcal{S U}_{E}(r, L)$ by Proposition 3.7 .

$$
\begin{aligned}
& \begin{array}{ccc}
\mathcal{S U}_{E}(r, L) & \cong & \mathbb{P}^{r-1} \\
W_{r, L}^{0}(\exists) & \cong & \mathbb{P}^{r-2} \\
\cup I & & \cup I
\end{array} \\
& W_{r, L}^{1}(\exists) \cong \mathbb{P}^{r-3} \\
& \text {. . } \\
& \text {. } \\
& \text { UI UI } \\
& \underset{\cup, L}{W_{r}^{r-3}(\exists) \cong \mathbb{P}^{1}} \\
& W_{r, L}^{r-2}(\exists) \cong \mathbb{P}^{0} \cong\{\cdot\}
\end{aligned}
$$

So, $W_{r, L}^{0}(\exists)$ is a subvariety of $\mathcal{S U}_{E}(r, L)$ of codimension 1 and hence a divisor. We can calculate relations between $\left[\mathbb{P}^{i}\right]^{\prime}$ 's as follows. Inside $\mathbb{P}^{r-1}$ we have the following stratification:

$$
\{\cdot\} \subseteq \mathbb{P}^{1} \subseteq \mathbb{P}^{2} \subseteq \cdots \subseteq \mathbb{P}^{r-2} \subseteq \mathbb{P}^{r-1}
$$

Then we have:

$$
H^{*}\left(\mathbb{P}^{r-1}, \mathbb{Z}\right)=\frac{\mathbb{Z}[\zeta]}{<\zeta^{r}>}
$$

where $\zeta$ is the cohomology class of $\mathbb{P}^{r-2}$, that is, $\zeta=\left[\mathbb{P}^{r-2}\right]=c_{1}(\mathcal{O}(1)), c_{1}(\mathcal{O}(1))$ being the first chern class of $\mathcal{O}(1)$ over $\mathbb{P}^{r-1}$. Moreover in $H^{*}\left(\mathbb{P}^{r-1}, \mathbb{Z}\right)$, we have:

$$
\left[\mathbb{P}^{r-1-k}\right]=\zeta^{k}
$$

Therefore, by (51) and Proposition 3.7 we get:

$$
H^{*}\left(\mathcal{S U}_{E}(r, L), \mathbb{Z}\right)=\frac{\mathbb{Z}[\zeta]}{<\zeta^{r}>}
$$

Furthermore, by (6) and Proposition 3.7 we get the following equality in $H^{*}\left(\mathcal{S U}_{E}(r, L), \mathbb{Z}\right)$ :

$$
\left[W_{r, L}^{i}(\exists)\right]=\left[\mathbb{P}^{r-i-2}\right]=\left[\mathbb{P}^{r-1-(i+1)}\right]=\zeta^{i+1}=\left[\mathbb{P}^{r-2}\right]^{i+1}=\left[W_{r, L}^{0}(\exists)\right]^{i+1} .
$$

Hence the theorem follows.

The next theorem is about some relations between the generators of the tautological subalgebra of $H^{*}\left(\mathcal{S U}_{E}(r, L), \mathbb{Z}\right)$ and $H^{*}\left(\mathcal{M}_{E}(r, 0), \mathbb{Z}\right)$. The theorem says that tautological algebra of $\mathcal{M}_{E}(r, 0)$ is generated by the cohomology class of the Brill-Noether subvariety $W_{r, 0}^{0}(\exists)$ as an $H^{*}(E)$-algebra.

Theorem 5.4. The tautological algebra of $\mathcal{M}_{E}(r, 0)$ is

$$
H^{*}(E) \otimes \mathbb{Z}(\xi) /\left(\xi^{r}\right)
$$

Here $\xi$ is the cohomology class of the divisor $W_{r, 0}^{0}(\exists)$ on $\mathcal{M}_{E}(r, 0)$ in $H^{*}\left(\mathcal{M}_{E}(r, 0), \mathbb{Z}\right)$. 
Proof. We have the following stratification inside $\mathcal{M}_{E}(r, 0)$ by Proposition 3.7.

$$
\begin{aligned}
& \mathcal{M}_{E}(r, 0) \cong S^{r} E \\
& \text { UI } \quad \text { I } \\
& W_{r, 0}^{0}(\exists) \cong S^{r-1} E \\
& \underset{\cup \mathrm{\cup l}}{W_{r, 0}^{1}(\exists) \cong} \stackrel{S^{r-2} E}{\cup \mid} \\
& \text {. . } \\
& \text {. } \\
& \text { UI UI } \\
& W_{r, 0}^{r-2}(\exists) \cong S^{1} E \cong E
\end{aligned}
$$

So, $W_{r, 0}^{0}(\exists)$ is a subvariety of $\mathcal{M}_{E}(r, 0)$ of codimension 1 and hence a divisor. By (33) and by Remark 4.1, the determinant morphism

$$
\mathcal{M}_{E}(r, 0) \rightarrow J(E)
$$

is a projective bundle $\mathbb{P}_{J(E)}^{r-1} \rightarrow J(E)$.

Hence, by projective bundle formula,

$$
H^{*}\left(\mathcal{M}_{E}(r, 0), \mathbb{Z}\right)=H^{*}(J(E)) \otimes \mathbb{Z}(\xi) /\left(\xi^{r}\right)
$$

Here $\xi$ is the first Chern class of $\mathcal{O}(1)$ on $\mathbb{P}_{J(E)}^{r-1}$.

Therefore by (17) and Proposition 3.7 we get,

$$
H^{*}\left(\mathcal{M}_{E}(r, 0), \mathbb{Z}\right)=H^{*}(E) \otimes \mathbb{Z}(\xi) /\left(\xi^{r}\right) .
$$

However, by (4), we have the equality of the cohomology classes:

$$
\left[W_{r, 0}^{i}(\exists)\right]=\xi^{i+1}
$$

for all $0 \leq i \leq r-2$. This gives the assertion.

\section{ACKNOWLEDGEMENT}

I would like to express my sincere gratitude to Prof. Jaya N. N. Iyer for introducing this problem to me and for her valuable suggestions. I would like to thank Dr. Archana S Morye and Dr. Tathagata Sengupta for their continuous guidance and encouragements throughout the work. I would also like to thank University Grants Commission (UGC) (ID - 424860) for financial support. 


\section{REFERENCES}

[1] E. Arbarello, M. Cornalba, P. A. Griffiths and J. Harris, Geometry of Algebraic Curves. Vol. I, Grundlehren der Mathematischen Wissenschaften [Fundamental Principles of Mathematical Sciences], Springer-Verlag, New York., 267 (1985).

[2] M. F. Atiyah, Vector bundles over an elliptic curve, Proc. London Math. Soc.(3), 7 (1957), 414-452.

[3] M. Bigas and L. Tu, Theta divisors for vector bundles, Contemp. Math., 136 (1992), 327-342.

[4] R. Donagi and L. Tu, Theta functions for $S L(n)$ versus $G L(n)$, Math. Res. Lett. 1, 3 (1994), 345-357.

[5] C. S. Seshadri, Space of unitary vector bundles on a compact Riemann surface, Ann. of. Math. (2), 85 (1967), 303-336.

[6] L. Tu, Semistable bundles over an elliptic curve, Adv. Math. 98, 1 (1993), 1-26.

School of Mathematics and Statistics, University of Hyderabad, Prof. C. R. Rao Road, P.O - Central University, Gachibowli, Hyderabad - 500046, India.

E-mail address: mukherjee90.arijit@gmail.com 